\title{
Revisão Sistemática sobre Jogos Digitais que utilizam Realidade Aumentada no Contexto Educacional Brasileiro
}

\author{
Cleber Silva de Oliveira ${ }^{1,2}$, Pollyana Notargiacomo Mustaro ${ }^{2}$ \\ ${ }^{1}$ Instituto Federal de Educação Ciência e Tecnologia de São Paulo (IFSP) - Campus \\ Guarulhos \\ Avenida Salgado Filho, 3501 - 07.115-000 - Guarulhos - SP - Brazil \\ ${ }^{2}$ Universidade Presbiteriana Mackenzie (UPM) \\ Rua da Consolação, 930 - 01.302-907 - São Paulo, SP - Brazil \\ cleber@ifsp.edu.br, pollyana.mustaro@mackenzie.br
}

\begin{abstract}
This paper analyzes, through a systematic review, as Brazilian researchers have made use of Augmented Reality (AR) in educational games, also called serious games. For this, the sample was made, by means of an automated tool, for papers published on the Brazilian Computer Society (SBC) event dedicated to video games and entertainment, SBGames, in the past eight years (2006-2013). Analysis of the results shows that the use of educational games in RA issue is present in the community, however, is still not explored in a representative manner, and it is necessary to link this discussion to the development, i.e., implementation of games with such characteristics.
\end{abstract}

Resumo. O presente artigo busca analisar, por meio de uma revisão sistemática, como os pesquisadores brasileiros têm feito uso de Realidade Aumentada (RA) em jogos educacionais, também denominados de serious games. Para isso, a amostra foi constituida, por meio de uma ferramenta automatizada, por artigos científicos publicados no evento da Sociedade Brasileira de Computação (SBC) dedicada a jogos eletrônicos e entretenimento, SBGames, nos últimos oito anos (2006-2013). A análise dos resultados aponta que a questão do uso de $R A$ em jogos educacionais encontra-se presente na comunidade, contudo, que ainda não é explorada de forma representativa, bem como que é necessário vincular esta discussão ao desenvolvimento, ou seja, à implementação de jogos com tais características.

\section{Introdução}

A disposição de objetos virtuais no mundo físico por meio de tecnologias, ou seja, a sua incorporação ao mundo real, é denominada de Realidade Aumentada (RA). Dentre as suas áreas de aplicação destacam-se o marketing, a propaganda, o treinamento militar, a saúde e o entretenimento, configurando um âmbito multidisciplinar [Furht 2011] que pode contribuir em diferentes áreas da educação formal por meio de sua combinação com jogos digitais.

Neste sentido, buscou-se, então, caracterizar as pesquisas brasileiras que tratem desta temática por meio da análise dos artigos de uso de jogos digitais com RA no contexto educacional publicados no evento com maior projeção nacional (e na América 
Latina) de jogos e entretenimento digital: o SBGames. Para tanto, foi realizada uma revisão sistemática que considerou a totalidade dos artigos publicados no portal geral (http://www.sbgames.org) deste evento da Sociedade Brasileira de Computação (SBC) e da Abragames (Associação Brasileira de Desenvolvedores de Jogos Digitais), configurando uma amostra que envolve os últimos oito anos de sua realização.

Os resultados indicam que o uso de RA associado a jogos digitais para educação ainda constitui um campo que requer maior exploração em território brasileiro. A investigação empreendida está organizada por meio dos seguintes tópicos: Referencial Teórico, que resgata conceitos pertinentes a presente pesquisa; Revisão Sistemática, que explora o conceito e função desta; Planejamento, que expõe as questões básicas da revisão sistemática; Materiais e Métodos, que descreve como foi realizada a coleta de dados e os critérios de inclusão/exclusão de artigos de acordo com o planejamento da revisão sistemática; Resultados Obtidos, que consolida os dados capturados e expõe informações gerais sobre o universo pesquisado; Jogos Digitais com RA no Contexto Educacional Brasileiro, que se propõe a responder às questões da revisão sistemática; e, finalmente, Considerações Finais consolida as conclusões do estudo e os percursos a serem empreendidos em novas investigações.

\section{Referencial Teórico}

A Realidade Aumentada (RA) é caracterizada pela apresentação de objetos virtuais sobrepostos ao ambiente do mundo físico, sendo que esta abordagem é realizada por meio de um dispositivo tecnológico que torna a visão do usuário "aumentada" [Azuma 1997], instituindo uma interação que não requer treinamento do usuário.

Por meio de sistemas de RA pode-se implementar experiências em que o estudante-usuário interage com ambientes e objetos tridimensionais mesclados com o ambiente real, instituindo uma construção de conhecimentos mediada por aspectos cognitivos relacionados às sensações, habilidades e emoções [Terashima 2001]. Tais interações ocorrem em tempo real, propiciando a efetiva participação dos estudantes, que deixam se atuarem como expectadores [Azuma 2001 e Stuart 2001].

Em relação à classificação dos sistemas de RA, esta possui quatro estruturas: visão ótica direta (óculos ou capacete), visão ótica por projeção (em que superfícies do mundo físico são usadas para a projeção de objetos virtuais), visão direta por vídeo (faz uso de câmera acoplada a capacete) e visão pautada em monitor (constituída por uma webcam que realiza a captura da cena do mundo físico para mistura-la aos objetos virtuais e apresentar o resultado em um monitor) [Azuma 2001].

Para a contextualização da RA em processos educacionais mediados por jogos eletrônicos é preciso conceituar jogos educacionais digitais. Estes são comumente denominados de serious games, sendo que tal denominação surgiu na década de 70 , quando Abt (1970) estabelece que tais artefatos não possuem restrição em relação à faixa etária, além de apresentarem flexibilidade para utilização em cenários educacionais distintos. Estas características contribuem para o desenvolvimento de habilidades pertinentes à tomada de decisão por meio do desempenho de papéis (numa arquitetura regida por regras e objetivos) e pela apresentação do feedback pertinente. À definição anterior acrescenta-se que serious games são aplicações computacionais interativas cujo objetivo é o de construir conhecimentos sobre temáticas diversificadas e 
desenvolver atitudes e/ou competências para performance em cenários reais [Bergeron 2006]. Contudo, cabe uma complementação feita por Klopfer et al. (2009), que caracterizam serious games como quaisquer videogames cuja a utilização possui uma finalidade educacional, independentemente de proposta de origem. Inclusive, Prensky (2001) destaca que os processos de interativos de aprendizagem baseada em jogos digitais (Digital Game Based Learning - DGBL) propiciam engajamento e ludicidade, colaborando para a apreensão de conteúdos complexos ou que apresentem dificuldade de assimilação ou aferição dos conhecimentos construídos, configurando "máquinas de aprendizado" [Gee 2003].

Portanto, jogos digitais com fins educacionais podem ser utilizados em diferentes graus (desde a educação básica até a Pós-Graduação) e apresentar temáticas relacionadas à aprendizagem de escrita, coordenação motora, saúde e mesmo simuladores de elementos físicos [Savi e Ulbricht 2008] ou envolver a aquisição de habilidades e competências profissionais [Shaffer 2008].

Outro aspecto a ser ressaltado refere-se à influência dos periféricos, bem como coerência para sua seleção, em relação à interface de controle em jogos digitais [Sodoswki Jr. e Stanney 2002]. Isso também refere-se ao tipo de representação do jogo, ou seja, um jogo 2D para ser representado em 2,5D, 3D, RV ou RA (caso do presente artigo), por exemplo, deve ser adaptado; da mesma forma, o mesmo critério deve ser usado nos casos em que um jogo é disponibilizado para plataformas distintas, que também podem implicar num tipo de interação diferenciada.

\section{Revisão Sistemática}

O conceito de revisão sistemática diz respeito à realização de uma revisão de literatura com parâmetros previamente planejados de forma sistemática, sendo que sua função é levantar e analisar estudos primários a partir de uma questão estabelecida com o intuito de constituir uma síntese [Gough, Oliver e Thomas 2012] e identificar áreas que requerem maiores estudos e podem ser empreendidas posteriormente [Kitchenham 2004].

Em relação à organização das atividades, Gough, Oliver e Thomas (2012) e Kitchenham (2004), instituem três blocos distintos para a realização da revisão sistemática:

1. Planejamento - envolve a delimitação do escopo, bem como a elaboração das questões que nortearão a revisão sistemática, além da construção e validação do protocolo de revisão;

2. Realização - distinção e seleção das pesquisas pertinentes, análise qualitativa dos documentos selecionados, bem como a organização e síntese de informações relevantes;

3. Documentação - construção de relatório que apresenta a estrutura e resultados da revisão sistemática realizada. 
A revisão sistemática comporta, também, a definição de questões, sendo que estas podem ser principais (QP) ou secundárias (QS). No caso do presente estudo as questões encontram-se listadas abaixo:

- QP1: São realizadas pesquisas no Brasil relacionadas à RA e educação?

- QP2: Há uma diferença numérica relevante entre a combinação de RV e educação e RA e educação?

- QS1: Os trabalhos apresentados possuem implementação ou tratam de aspectos teóricos?

Com base na estrutura mapeada anteriormente foi estabelecida a amostra, formada pelo conjunto de artigos que satisfazem as premissas de inclusão, bem como desenvolvidos algoritmos que facilitassem e automatizassem elementos específicos do processo, sendo os mesmos descritos a seguir.

\section{Materiais e Métodos}

Para a realização da presente revisão sistemática optou-se pelo estudo de pesquisas na área de jogos digitais, divulgadas em âmbito nacional por meio do SBGames. Este é o maior evento brasileiro na área de jogos e entretenimento digital, bem como o principal fórum acadêmico de investigações e desenvolvimento de jogos na América Latina, integrando a comunidade acadêmica, de desenvolvimento e da indústria. Este evento da Sociedade Brasileira de Computação (SBC) e da Abragames (Associação Brasileira dos Desenvolvedores de Jogos Digitais) possui quatro Trilhas (Computação, Arte \& Design, Cultura e Indústria) distribuídas em Sessões Técnicas, em que são apresentados trabalhos completos (full papers) e pôsteres (short papers). A primeira edição do evento ocorreu em 2002, sendo que a partir do ano de 2006 os anais passaram a ser publicados em formato eletrônico.

Para a presente investigação foi necessária a aquisição dos documentos pertinentes aos Anais Eletrônicos do SBGames no período de 2006 a 2013. Para isso foi desenvolvida uma ferramenta automatizada de extração dos dados para análise, sendo que os mesmos (após o processo de extração nos documentos em PDF) foram convertidos em texto puro e armazenados numa base de dados. Essa abordagem foi adotada porque nos anos anteriores os anais foram impressos, impedindo seu acesso via web.

Assim, se trabalhou com a totalidade dos artigos publicados em oito edições do evento, o que totalizou 872 artigos. Em seguida foi desenvolvida, utilizando-se a linguagem Java e o Apache Lucene (um motor de busca e indexação de textos), uma aplicação para extração e busca de palavras-chave na base de artigos adquirida. Segundo Milosavljevic, Boberic e Surla (2010), usar o motor de busca Apache Lucene facilita a construção de sistemas de recuperação altamente flexíveis e eficientes. Inclusive, o Apache Lucene seria utilizado integralmente para a extração e busca de palavras-chave, porém um pacote interno do Lucene chamado Apache PDFBox, responsável pela conversão de arquivos em formato PDF para TXT, gerava alguns erros em parte dos documentos. Como a plataforma utilizada foi o Linux, aproveitou-se uma ferramenta de console chamada "poppler-utils", pois já encontra-se presente nesta o comando 
"pdftotxt" (comando padrão que poderia percorrer todos os documentos pelo shell de comandos do Linux).

Portanto, foram observados que os erros tornavam-se recorrentes e ainda assim, 15 artigos apresentaram problemas para a extração e/ou conversão, sendo que as informações pertinentes aos mesmos (título, autores, instituições, abstract) tiveram que ser extraídas manualmente.

Dentre os problemas enfrentados destaca-se a proteção de documentos (que inviabilizou a cópia dos caracteres presentes nos mesmos, resultando em textos sem sentido, como por exemplo, “-/ )1)@N)LB)KLLM-)4-”). Outro problema documentado durante a extração dos dados refere-se ao erro de charset para a conversão de caracteres com acentuação, implicando na necessidade de tratamento de dados dos caracteres "ç", " ", "'" e "^". Tendo em vista tais questões, a solução aplicada para comparação dos dados envolveu converte-los para letras minúsculas e retirar toda acentuação, garantindo que não ocorram problemas de case-sensitive (diferenciação entre letras maiúsculas e minúsculas) e charset (tipos de caracteres diferentes na representação ASCII) diferentes.

Cabe ressaltar também que, a partir das questões (estabelecida na configuração da revisão sistemática) se trabalhou com os seguintes termos: "virtual reality", "realidade virtual", "augmented reality", "realidade aumentada", "education" e "educação".

Como se trata de um busca léxica de comparação de termos, reduziu-se o número de palavras utilizando uma lista de "stop words" (palavras que podem desconsideradas) em inglês e português, já que os artigos podem apresentar ambas línguas ou apenas uma delas. Assim, o desempenho do algoritmo de busca deu-se em $\mathrm{O}(\mathrm{n})$, onde as palavras presentes na base de artigos só foram percorrida uma vez e comparadas com os termos.

\section{Resultados Obtidos}

Após a coleta automatizada dos dados, obteve-se a seguinte tabela (Tabela 1).

Tabela 1. Consolidação dos artigos da revisão sistemática classificados pelos termos de ocorrência

\begin{tabular}{|l|l|}
\hline Classificação & Número de Artigos \\
\hline Nenhum Termo & 455 \\
\hline Somente RV & 82 \\
\hline Somente RA & $17\left(19^{*}\right)$ \\
\hline Somente Educação & 235 \\
\hline RV e RA & 23 \\
\hline RV e Educação & 40 \\
\hline RA e Educação & $7\left(5^{\star}\right)$ \\
\hline RV e RA e Educação & 13 \\
\hline TOTAL & 872 \\
\hline
\end{tabular}

* Dois artigos [Paula, Bonini Neto e Miranda 2006] e [Macedo e Rodrigues 2012] foram excluídas da classificação "RA e Educação" durante a revisão sistemática, pois apresentam o termo "education" nas referências, mas são trabalhos somente sobre RA. 
Em primeiro lugar, cabe destacar que aproximadamente $52,18 \%$, ou seja, mais da metade da amostra total não possui relação com nenhum dos termos encontrados. Isso pode ser explicado pela própria natureza do evento SBGames, já que o mesmo abarca quatro áreas (computação, arte \& design, cultura e indústria). Ainda assim, mais de $25 \%$ do universo pesquisado relaciona-se ao âmbito educacional, mostrando que os jogos digitais podem colaborar em processos de aprendizagem [Gee 2003, Abt 1970, Prensky 2001].

No que se refere à área de RV e RA, ressalta-se que ambas ainda não possuem representatividade nas investigações veiculadas em âmbito nacional por meio do evento SBGames. Neste sentido, apenas 9,4\%, ou seja, menos de $10 \%$ dos artigos que constituem a amostra do presente estudo, relaciona-se de alguma maneira à $R V, 2 \%$ à RA e 2,6\% à RV e RA. Quando se trata da combinação de RV ou de RA (ou de ambas) aos processos educacionais, este universo torna-se ainda mais restrito, sendo que aproximadamente $4,6 \%$ da amostra combina $R V$ à educação e somente aproximadamente $0,6 \%$ relaciona RA è educação - a combinação de ambas à educação resulta num percentual de aproximadamente 1,5\%. Assim, percebe-se que a utilização de RV e, sobretudo, de RA para a construção de conhecimentos mediados tecnologicamente ainda constitui uma área a ser explorada mais pelos cientistas no Brasil.

\section{Jogos Digitais com RA no Contexto Educacional Brasileiro}

Após o levantamento e categorização dos artigos do SBGames passou-se, então, à análise dos documentos que, pela classificação automatizada apresentavam conjuntamente os termos RA e educação (ou seus correlatos, conforme descrito nos materiais e métodos) para responder às questões da revisão sistemática. A lista consolidada dos artigos que envolvem RA em processos educacionais encontra-se abaixo, na Tabela 2.

Tabela 2. Lista de artigos publicados que fazem uso de RA em jogos educacionais

\begin{tabular}{|l|l|}
\hline Título & Autores \\
\hline $\begin{array}{l}\text { enJine: Architecture and Application of an Open-Source } \\
\text { Didactic Game Engine }\end{array}$ & [Nakamura, Bernardes e Tori 2006] \\
\hline Coleta Seletiva: Educação ambiental com webcam game & [Souza Jr., Ribeiro e Dazzi 2009] \\
\hline A mágica do jogo e o potencial do brincar & $\begin{array}{l}\text { [Brandão, Bittencourt e Vilhena } \\
\text { 2010] }\end{array}$ \\
\hline $\begin{array}{l}\text { What is going on with ubicomp games } \\
\text { Coletando: Desenvolvimento de um jogo para o ensino- } \\
\text { aprendizagem no campo da Educação Ambiental }\end{array}$ & [Buzeto, Castillo e Castanho 2012] \\
\hline
\end{tabular}

De acordo com a Tabela 2 pode-se dizer que existe, ainda que numa escala reduzida, pesquisas brasileiras voltadas ao uso de RA em jogos digitais concernentes a processos educacionais. As mesmas encontram-se descritas e analisadas a seguir.

O primeiro trabalho a fazer menção à questão de RA e Educação, mapeado por esta revisão sistemática, é o artigo enJine: Architecture and Application of na OpenSource Didact Game Engine de Nakamura, Bernardes e Tori (2006). Neste é apresentada a engine (motor de jogos) Open-Source desenvolvida na POLI-USP com Java 3D API. Cabe destacar que o trabalho ressalta a possibilidade de desenvolvimento 
de jogos com realidade virtual e aumentada, bem como a estrutura que suporta a elaboração de jogos educacionais.

Já o artigo A mágica do jogo e o potencial de brincar - de Brandão, Bittencourt e Vilhena (2010) - trata das potencialidades dos jogos digitais que transcendem a diversão. Assim, é realizada uma discussão sobre o brincar e o ludus, além de explorar serious games [Abt 1970, Prensky 2001]. Em relação à realidade aumentada, são apresentados, como exemplos, o Webkinz (jogo online para PC) e EyePet (para Playstation 3).

A proposta do trabalho What is going on with ubicomp games [Buzeto, Castillo e Castanho 2012] volta-se ao levantamento de características para o design de ubigames (jogos que envolvem computação ubíqua) e realiza uma revisão de 30 artigos no período de 2002 a 2011. Ao longo da revisão é que são citadas as questões da realidade aumentada e educação, apresentando potencialidades em relação aos jogos com computação ubíqua.

$\mathrm{O}$ artigo Coletando: Desenvolvimento de um jogo para o ensino-aprendizagem no campo da Educação Ambiental [Andrade et al. 2012] apresenta um jogo educacional sobre coleta seletiva de lixo. A questão da realidade aumentada é citada nos trabalhos relacionados ao descrever um jogo de coleta seletiva para crianças de 4 a 6 anos [Souza Jr., Ribeiro e Dazzi 2009] em que o movimento das mãos (capturada por uma webcam) é utilizado para solucionar a questão vinculada à coordenação motora (dificuldade de uso de joystick para crianças desta faixa etária), estando em conformidade com o que é apontado por Sodoswki Jr. e Stanney (2002) e por Bergeron (2006) em relação ao controle do jogo e ao desenvolvimento de atitudes por meio de jogos digitais, respectivamente.

Finalmente, o trabalho Coleta Seletiva: Educação ambiental com webcam game [Souza Jr., Ribeiro e Dazzi 2009], conforme citado no parágrafo anterior, aborda a implementação de um jogo educacional de realidade aumentada com webcam para coleta seletiva (visão pautada em monitor [Azuma 2001]), instituindo uma mecânica para a coleta de lixo virtual com movimentação das mãos e corpo. Dentre os resultados, os autores destacam a facilidade de adaptação e concentração por parte das crianças, bem como a naturalidade para a interação. Destaca-se que a proposta está de acordo com o que é colocado por Terashima (2001), em relação ao uso de RA na educação, e com Azuma (2001) e Stuart (2001) no que se refere à postura ativa do estudante-usuário.

\section{Considerações Finais}

Identificou-se que são realizadas investigações envolvendo o uso de RA em jogos digitais de cunho educacional (QP1). Também foi possível perceber que existe uma diferença numérica quando se trata de RV e educação e RA e educação (QP2) e que os trabalhos apontam possibilidades de uso de RA no âmbito educacional por meio de jogos, contudo, somente um artigo analisado possui implementação (QS1), configurando um campo de pesquisa que pode ser ampliado no cenário nacional.

Dentre os trabalhos futuros destaca-se a análise dos trabalhos acadêmicos presentes no SBIE (Simpósio Brasileiro de Informática na Educação) e, posteriormente, no CBIE (Congresso Brasileiro de Informática na Educação), dado que este é o maior 
evento nacional da SBC dedicado à discussão da utilização de tecnologias digitais (inclusive jogos) em situações de aprendizagem, bem como explorar os artigos publicados no SVR (Symposium on Virtual and Augmented Reality), evento também vinculado à SBC. Pretende-se, ainda, realizar um estudo bibliométrico e cientométrico, atentando para o estabelecimento de uma rede de coautoria dos artigos publicados no SBGames e, em seguida realizar a mesma abordagem no contexto do SBIE, CBIE e SVR. Com isso se buscará identificar os autores e referências mais relevantes para a comunidade de pesquisadores brasileiros, bem como verificar geograficamente onde se encontram diferentes expertises nesta área de pesquisa.

Também se pretende realizar um estudo comparativo da revisão sistemática circunscrita ao SBGames com eventos internacionais como o DiGRA.

De forma complementar, também, devido aos problemas para a extração automatizada dos dados apresentados, se buscará melhorar os algoritmos de identificação.

\section{Agradecimentos}

Os autores agradecem à Universidade Presbiteriana Mackenzie e ao MackPesquisa pelo suporte disponibilizado para a realização da investigação.

\section{Referências}

ABT, C. (1970). Serious Games. Viking Press, New York.

Andrade, M., Almeida, E., Oliveira, T., Silva, C., Falcão, F., Melo, M. T. e Araújo, A. (2012). Coletando: Desenvolvimento de um jogo para o ensino-aprendizagem no campo da Educação Ambiental. In: Proceedings of SBGames 2012, p. 188-191, Brasília, SBC.

Azuma, R. T. (1997). “A Survey of Augmented Reality”, In Presence: Teleoperators and Virtual Environment, v. 6, n. 4, p. 355-385.

Azuma, R. et al. (2001). Recents Advances in Augmented Reality. In IEEE Computer Graphics and Applications, vol. 21, p. 34-37.

Brandão, R. P., Bittencourt, M. I. F. Vilhena, J. (2010). A mágica do jogo e o potencial do brincar. In: Proceedings do SBGames 2010, Trilha de Games \& Cultura - Full Papers, p. 11-20, SBC.

Bergeron, B. P. (2006). Developing Serious Games. Thomson Delmar Learning / Charles River Media, Inc., Massachusetts.

Buzeto, F. N., Castillo, A. H. O. R. e Castanho, C. D. (2012). What is going on with ubicomp games. In: Proceedings of SBGames 2012, Computing Track - Full Papers,

1-7, Brasília, SBC.

Furht B. (2011). Augmented Reality: An Overview. In: Carmigniani J., Furht B. (Eds). Handbook of Augmented Reality. New York: Springer, 2011.

Gee, J. P. (2003). What video games have to teach us about learning and literacy. Palgrave/Macmillan, New York. 
Gough D, Oliver S, Thomas J. An introduction to Systematic Reviews. SAGE Publications, 2012.

Kitchenham B. Procedures for Performing Systematic Reviews. Technical Report TR/SE0401, Keele University, and Technical Report 0400011T.1, National ICT Australia, 2004.

Klopfer, E. et al. (2009). From Serious Games to Serious Gaming. In: Ritterfeld, U., Cody, M. e Vorderer, P. (Eds). Serious Games: Mechanisms and Effects. Routledge, New York.

Macedo, D. V. e Rodrigues, M. A. F. (2012). Experiências com Desenvolvimento Ágil de um Jogo Casual para Plataformas Móveis usando o Motor Gráfico Unity. In: Proceedings of SBGames 2012, p. 148-156, Brasília, SBC.

Milosavljevic , B., Boberic, D. e Surla, D. (2010). Retrieval of bibliographic records using Apache Lucene. The Electronic Library, 525-539.

Nakamura, R. Bernardes e Tori, R. (2006). enJine: Architecture and Application of an Open-Source Didactic Game Engine. In: Digital Proceedings of the V Brazilian Symposium on Conputer Games and Digital Entertainment, p. 1-7, Recife, SBC.

Paula, L. R. P., Bonini Neto, R. e Miranda, F. R. (2006). Digital Proceedings of the V Brazilian Symposium on Conputer Games and Digital Entertainment, p. 1-9, Recife, SBC.

Prensky, M. (2001). Digital Game Based Learning. McGraw-Hill, New York.

Savi, R. e Ulbricht, V. R. (2008). Jogos digitais educacionais: benefícios e desafios. In Novas Tecnologias na Educação, v. 6, n. 2, p. 1-10, CONTED-UFRGS.

Shaffer, D. W. (2008). How Computer Games Help Children Learn. Palgrave Macmillan.

Sodowski Jr., W. e Stanney, K. (2002). Chapter 45: Measuring and Managing Presence in Virtual Environments [online] In: Stanney, K. (Ed.). Handbook of virtual environments: Design, implementation, and applications. 2002. http://vehand.engr.ucf.edu/handbook/.

Souza Jr., E., Ribeiro, N. E. C. e Dazzi, R. L. S. (2009). Coleta Seletiva: Educação ambiental com webcam game. In: VIII Brazilian Symposium on Games and Digital Entertainment, p. 77-82, Rio de Janeiro, SBC.

Stuart, R. (2001). The Design of Virtual Environments. Barricade Books Inc., Canada.

Terashima, N. (2001). The Definition of Hyperreality. In: Tiffin, J. e Terashima, N. Hyperreality: Paradigm for the third millennium. Routledge, New York, p.4-24. 